\title{
Comparison of e-test Values for Standard Antibiotics and Conventional Antimicrobial Assay Values for Ethanoic Acids against Nosocomial Multidrug- resistant Pseudomonas aeruginosa
}

\author{
Muazzam Sheriff Maqbul' ${ }^{1}$, Ali Mohamed Alshabi², Aejaz A. Khan³, S.M. Shakeel \\ Iqubal $^{3 *}$ (D), Tasneem Mohammed ${ }^{3}$, Ibrahim Ahmed Shaikh ${ }^{4}$, Areej Dawoud ${ }^{3}$, \\ Uday M. Muddapur ${ }^{5}$, Mohammed Shahid Hussain ${ }^{6}$ and S.K. Singh ${ }^{7}$ \\ ${ }^{1}$ Faculty of Microbiology and Immunology, Ibn Sina National College of Medical Sciences, Al Mahjar Street: 31906, \\ Jeddah 21418, Kingdom of Saudi Arabia. ${ }^{2}$ Department of Clinical Pharmacy, College of Pharmacy, Najran University, \\ Najran, Saudi Arabia. ${ }^{3}$ Department of General Science, Ibn Sina National College of Medical Sciences, Al Mahajar \\ Street: 31906, Jeddah 21418, Kingdom of Saudi Arabia. ${ }^{4}$ Department of Pharmacology, College of Pharmacy, Najran \\ University, Najran, Saudi Arabia. ${ }^{5}$ Department of Biotechnology, KLE Technological University, BVB Campus, Hubballi, \\ 580031, India. ${ }^{6}$ Department of Orthodontics and Dentofacial Orthopedics, M. A. Rangoonwala Dental College, \\ Pune, Maharashtra, India. 'Department of Chemistry, GGV (Central University), Bilaspur (C.G) - 495009 , India.
}

\begin{abstract}
The present study aimed to determine the susceptibility of Pseudomonas aeruginosa strains isolated from patients with nosocomial infections to standard synthetic chemical antibiotics and organic ethanoic acids derived from local produce. The minimum inhibitory concentrations (MIC) of the standard synthetic antibiotics determined from standard e-test results and antibiotic sensitivity tests showed many multidrug-resistant strains among the isolates. We compared the susceptibility of these strains to organic ethanoic acids derived from different sources using standard microbiological assays. All strains of $P$. aeruginosa isolated from the patients were susceptible to the organic ethanoic acids with a satisfactory MIC and minimum bactericidal concentrations. Therefore, organic ethanoic acids were more effective against $P$. aeruginosa than standard synthetic antibiotics.
\end{abstract}

Keywords: Antimicrobial activity, Pseudomonas aeroginosa, ethanoic acid, Nosocomial infection, Immuno suppressed, Vinegar

\footnotetext{
*Correspondence: shakeeliqubal@gmail.com; +966-570158198
}

(Received: December 13, 2019; accepted: March 18, 2020)

Citation: Muazzam Sheriff Maqbul, Ali Mohamed Alshabi, Aejaz A. Khan, S.M. Shakeel Iqubal, Tasneem Mohammed, Ibrahim Ahmed Shaikh, Areej Dawoud, Uday M. Muddapur, Mohammed Shahid Hussain and S.K. Singh, Comparison of e-test Values for Standard Antibiotics and Conventional Antimicrobial Assay Values for Ethanoic Acids against Nosocomial Multidrug-resistant Pseudomonas aeruginosa, J. Pure Appl. Microbiol., 2020; 14(1):255-260. https://doi.org/10.22207/JPAM.14.1.26

(C) The Author(s) 2020. Open Access. This article is distributed under the terms of the Creative Commons Attribution 4.0 International License which permits unrestricted use, sharing, distribution, and reproduction in any medium, provided you give appropriate credit to the original author(s) and the source, provide a link to the Creative Commons license, and indicate if changes were made. 


\section{INTRODUCTION}

Pseudomonas aeruginosa is a Gramnegative motile bacterium that is a major cause of community-acquired, and hospital-borne nosocomial infections $\mathbf{s}^{1,2}$. The major concern about nosocomial infections with $P$. aeruginosa is that $>10 \%$ of infections are caused by multidrugresistant species ${ }^{1,3,4}$ that arise through antibiotic abuse. Pseudomonas is only one among many bacterial species that have become resistant to multiple antibiotics, and the most resistant strain is $P$. aeruginosa $a^{5}$. Gessard isolated $P$. aeruginosa from green pus in 1882 and since then it has been studied in detail, especially with regard to nosocomial infections ${ }^{1}$. The characteristic virulence factors of this bacterium are the exopigments pyoverdin and pyocyanin, which are potentially involved in the occurrence of these infections ${ }^{1}$. Pseudomonas aeruginosa causes versatile infections in humans, especially when immunosuppressed, and it is categorized as the most important bacterial cause of infections acquired during prolonged hospitalization. This bacterium causes frequent infections due to its natural adaptability and abundance, and it has emerged as the most important species with which to analyze multidrug resistance ${ }^{1,6}$. Here, we isolated multidrug-resistant strains from clinical specimens derived from patients with nosocomial infections, then compared the susceptibility of these strains to commercial synthetic antibiotics and to ethanoic acids prepared from organic apple cider, sugar molasses, dates, grapes, and grains using conventional standard microbiological techniques $s^{4,7,8,9}$. Pseudomonas aeruginosa ATCC27853 was the standard control strain. Because ethanoic acids contains carboxylic acids that control the formation of $P$. aeruginosa biofilm, they should help to suppress infection with this bacterium ${ }^{6,8,9}$.

\section{MATERIALS AND METHODS}

All chemicals, reagents, and media components for this study were purchased from Sd Fine Chem Ltd, (Kolkata, India) Loba Chemie PvT., Ltd. (Mumbai, India), HiMedia (Mumbai, India), bioMérieux SA., (Marcy l'Etoile, France or Sigma Aldrich Corp. (St. Louis MO, USA).

\section{Isolation and purification of $\boldsymbol{P}$. aeruginosa}

Green pus, green catheters, urine, greenish ventilators, abscesses, throat swabs, nasal swabs, aspirates and collected from patients with nosocomial infections, and samples collected at surgery were processed using standard aseptic microbiological techniques. The samples were streaked onto plates containing cetrimide agar, a selective and differential medium, and incubated overnight at $37^{\circ} \mathrm{C}$ to allow $P$. aeruginosa to secrete exopigments. The isolates were identified and purified based on positive oxidase tests. The authenticity of the standard $P$. aeruginosa ATCC 27853 strain was confirmed in the same manner ${ }^{5,10,11}$.

\section{Antimicrobial susceptibility tests}

The antimicrobial susceptibility of the clinical isolates and the standard strain to standard synthetic chemical antibiotics was assessed using rapid e-tests ${ }^{4,5,7}$, Briefly, the isolates were inoculated onto separate plates containing Mueller-Hinton agar and standard e-test plastic strips were infused with each antibiotic and incubated with the isolates at $37^{\circ} \mathrm{C}$ overnight to develop zones and ellipses. The interaction of the ellipse was taken as the minimum inhibitory concentration (MIC), whereas the zone indicated the susceptibility of the antibiotic to the bacterium. Conventional standard was employed to observe The susceptibility of the $P$. aeruginosa isolates and the standard strain to five organic ethanoic acids was assessed by the standard diffusion method using Kirby-Bauer discs. Briefly, the isolates were inoculated separately on Mueller-Hinton agar plates with the infused discs for $24 \mathrm{~h}$ at $37^{\circ} \mathrm{C}$ to form zones, indicating the sensitivity of the bacterium toward the organic ethanoic acids. The MIC and minimum bactericidal concentration (MBC) reflecting the ability of ethanoic acid to kill the bacterium were estimated using the standard tube dilution method. Briefly, the isolates were separately inoculated into ethanoic acids diluted in peptone water, and incubated for $24 \mathrm{~h}$ at $37^{\circ} \mathrm{C}$. The absence of turbidity indicated the sensitivity of the bacterium to the test agent. The last dilution with turbidity determined the MIC value of the test agent against the bacterium.

The MBC was determined by inoculating each dilution of MIC onto separate agar plates 
for each isolate $e^{5,10,11,12}$, then incubating them for $24 \mathrm{~h}$ at $37^{\circ} \mathrm{C}$. The first dilution with no growth defined the $\mathrm{MBC}$ of the acid toward the bacterium Pseudomonas aeruginosa ATCC278.

\section{RESULTS AND DISCUSSION}

The results obtained from the e-tests of the susceptibility of $P$. aeruginosa to standard synthetic chemical antibiotics compared with organic ethanoic acid showed that none of the clinical $P$. aeruginosa isolates were resistant to organic ethanoic acid, whereas all the clinical isolates were resistant all the standard synthetic antibiotics tested. The best and worst antibiotic zone diameters for the standard synthetic chemical antibiotics against the clinical isolates

Table 1. Comparison of Pseudomonas aeruginosa susceptibility to standard antibiotics according to e-test zone diameters $(\mathrm{mm})$.

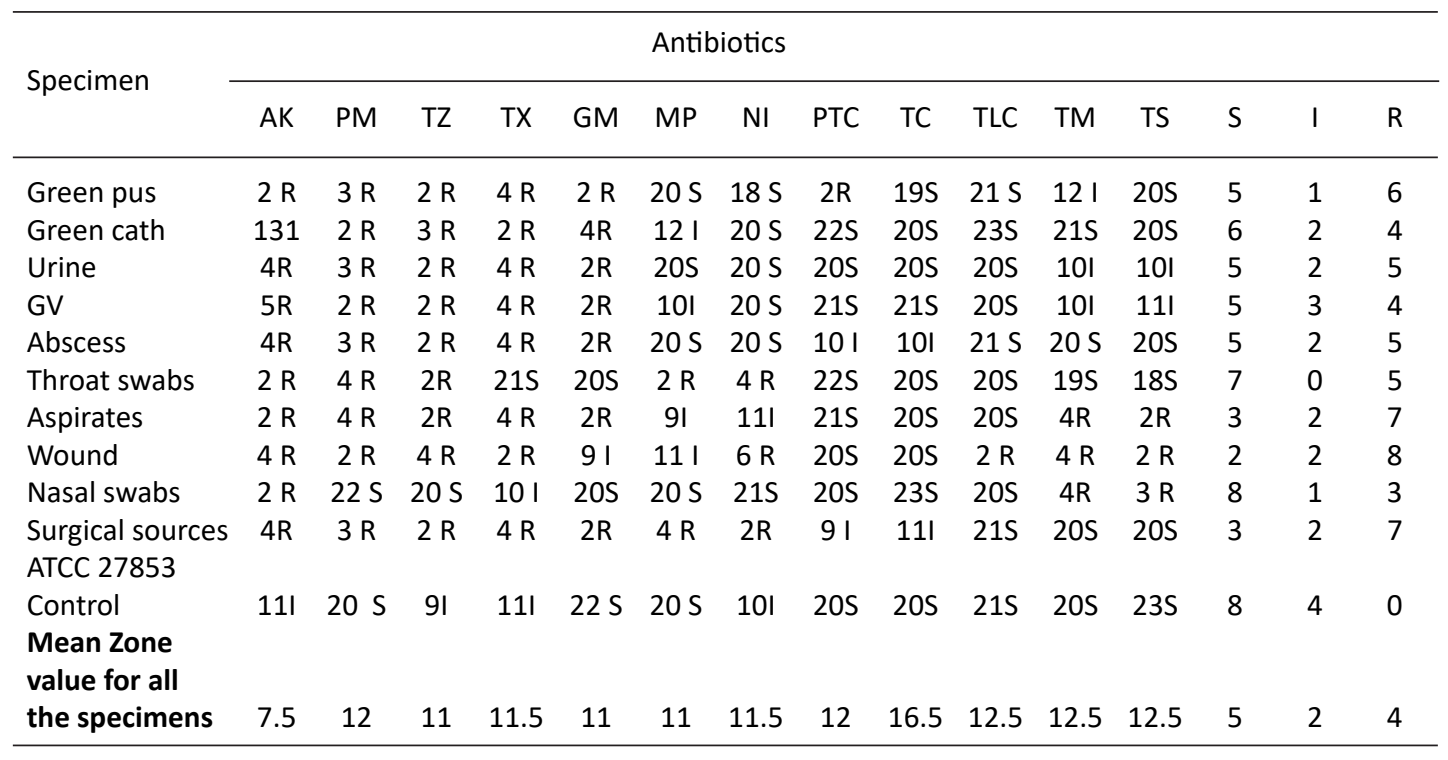

I, intermediate susceptibility; R, resistant; S, susceptible.

Table 2. Comparison of Pseudomonas aeruginosa susceptibility to standard antibiotics determined as minimum inhibitory concentrations in e-tests.

\begin{tabular}{lcccccccccccc}
\hline & \multicolumn{10}{c}{ Antibiotic MIC $(\mu \mathrm{g} / \mathrm{mL})$} \\
\cline { 2 - 6 } Specimen & AK & PM & TZ & TX & GM & MP & NI & PTC & TC & TLC & TM & TS \\
\hline Green pus & 1 & 1 & 1 & 0.25 & 0.25 & 0.5 & 0.5 & 0.5 & 0.25 & 0.25 & 0.5 & 0.5 \\
Green cath & 1.25 & 0.5 & 1 & 1 & 0.25 & 0.5 & 0.5 & 0.5 & 0.25 & 0.25 & 0.25 & 0.5 \\
Urine & 1 & 1 & 1 & 0.25 & 0.25 & 0.5 & 1 & 1 & 1 & 0.5 & 0.25 & 0.5 \\
GV & 1 & 1 & 1 & 0.25 & 0.25 & 0.5 & 0.5 & 0.25 & 0.25 & 0.25 & 0.25 & 0.5 \\
Abscess & 1 & 1 & 1 & 0.25 & 0.25 & 0.5 & 1 & 1 & 1 & 0.25 & 0.25 & 0.25 \\
Throat swab & 0.25 & 0.25 & 0.5 & 1 & 1 & 0.25 & 1 & 1 & 1 & 0.5 & 0.5 & 0.25 \\
Aspirates & 0.25 & 0.5 & 0.5 & 0.5 & 0.25 & 0.5 & 0.5 & 0.5 & 0.25 & 0.25 & 0.5 & 0.5 \\
Wound & 1 & 1 & 1 & 0.25 & 0.25 & 0.5 & 0.25 & 0.5 & 0.5 & 0.5 & 0.25 & 0.5 \\
Nasal swab & 0.25 & 0.25 & 0.5 & 1 & 1 & 1 & 1 & 1 & 0.25 & 0.25 & 0.25 & 0.25 \\
Surgical & 0.25 & 0.5 & 0.5 & 0.5 & 0.25 & 0.25 & 0.5 & 0.5 & 0.5 & 0.25 & 0.5 & 0.25 \\
ATCC 27853 & 0.25 & 0.5 & 0.5 & 0.5 & 0.25 & 0.25 & 0.5 & 0.5 & 0.25 & 0.25 & 0.25 & 0.25 \\
Mean MIC* & 0.75 & 0.625 & 0.75 & 0.625 & 0.625 & 0.625 & 0.625 & 0.625 & 0.625 & 0.375 & 0.375 & 0.375 \\
\hline
\end{tabular}

*For all samples. Cath, catheter; GV, greenish ventilator; MIC, minimum inhibitory concentrations $(\mu \mathrm{g} / \mathrm{mL})$; Surgical, sourced during surgery. 
of $P$. aeruginosa were 23 and $2 \mathrm{~mm}$, respectively, compared with 29 and $20 \mathrm{~mm}$, respectively, for organic ethanoic acids. Among the 10 clinical isolates, the strain from the wound sample was resistant to 8 of the 12 standard synthetic chemical antibiotics, and sensitive to piperacillin/ tazobactam (PTC) and tetracycline (TC) with a zone diameter of $20 \mathrm{~mm}$. This strain also had intermediate sensitivity toward gentamicin (GM) and meropenem (MP) with zone diameters of 9 and $11 \mathrm{~mm}$, respectively. The clinical $P$. aeruginosa isolates from the wound sample were the most multidrug-resistant among the strains isolated herein, with different degrees of sensitivity to all tested antimicrobial agents. These results showed that organic ethanoic acids were more effective than standard synthetic antibiotics even against the most multidrug-resistant of the clinical $P$. aeruginosa isolates obtained herein.

Tables 1 and 3 show detailed comparison of the antimicrobial susceptibilities of all $P$. aeruginosa isolates from patients with nosocomial infections to standard synthetic chemical antibiotics and organic ethanoic acids. The mean MIC of standard antibiotic dilutions in e-tests and of organic ethanoic acids ranged from 0.375 $0.750 \mu \mathrm{L} / \mathrm{mL}$ and $0.25-0.875 \mu \mathrm{L} / \mathrm{mL}$, respectively for all $P$. aeruginosa isolates tested. Tables 2 and 4 respectively show details of the MIC of the standard synthetic chemical antibiotics and organic ethanoic acids for all Pseudomonas aeruginosa isolates from patients with nosocomial infections. The mean values of the MBC of organic ethanoic acid ranged from $0.5-1.25 \mu \mathrm{L} / \mathrm{mL}$ toward all

Table 3. Comparison of diffusion zone diameters $(\mathrm{mm})$ among Pseudomonas aeruginosa isolates that were susceptible to organic ethanoic acids in conventional assays.

\begin{tabular}{lccccc}
\hline \multirow{2}{*}{ Sample } & \multicolumn{5}{c}{ Organic ethanoic acid sources } \\
\cline { 2 - 5 } & Apple cider & Sugar molasses & Dates & Grapes & Grains \\
\hline Green pus & 22 & 22 & 22 & 22 & 22 \\
Green catheter & 20 & 22 & 19 & 22 & 22 \\
Urine & 21 & 21 & 20 & 22 & 24 \\
Greenish ventilator & 22 & 22 & 23 & 22 & 23 \\
Abscess & 27 & 22 & 24 & 22 & 24 \\
Throat swab & 24 & 22 & 23 & 26 & 26 \\
Aspirates & 23 & 26 & 24 & 22 & 22 \\
Wound & 24 & 22 & 22 & 22 & 22 \\
Nasal swab & 22 & 22 & 22 & 22 & 22 \\
Surgical samples & 25 & 22 & 22 & 22 & 22 \\
ATCC 27853 (Control) & 29 & 26 & 26 & 21 & 20 \\
Mean Zone diameter (mm) & 24.5 & 24 & 21.5 & 23.5 & 23 \\
\hline
\end{tabular}

Table 4. Comparison of minimum inhibitory concentrations of organic ethanoic acids among Pseudomonas aeruginosa isolates.

\begin{tabular}{lccccc}
\hline \multirow{5}{*}{ Sample } & \multicolumn{5}{c}{ Organic ethanoic acids $(\mu \mathrm{g} / \mathrm{mL})$} \\
\cline { 2 - 5 } & Apple cider & Sugar molasses & Dates & Grapes & Grains \\
\hline Green pus & 0.25 & 0.5 & 1 & 1 & 1 \\
Green catheter & 0.25 & 0.25 & 1 & 1 & 0.75 \\
Urine & 0.25 & 0.5 & 1 & 0.75 & 1 \\
Greenish ventilator & 0.25 & 0.25 & 1 & 0.5 & 1 \\
Abscess & 0.25 & 0.5 & 0.75 & 0.5 & 0.75 \\
Throat swabs & 0.25 & 0.25 & 0.5 & 0.5 & 1 \\
Aspirates & 0.25 & 0.75 & 0.75 & 1 & 0.5 \\
Wounds & 0.25 & 0.75 & 0.75 & 0.75 & 0.5 \\
Nasal swab & 0.25 & 0.25 & 1 & 0.5 & 1 \\
Surgical sources & 0.25 & 0.75 & 1.25 & 1 & 0.5 \\
ATCC 27853 & 0.25 & 0.25 & 0.5 & 0.5 & 0.5 \\
Mean MIC $(\mu \mathrm{g} / \mathrm{mL}$ ) & 0.25 & 0.5 & 0.875 & 0.75 & 0.75 \\
\hline
\end{tabular}

MIC, minimum inhibitory concentrations. *Derived from all samples. 


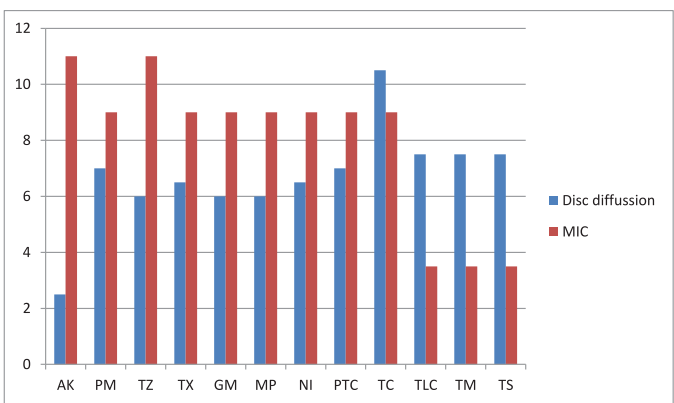

Fig. 1. Comparison of mean e-test susceptibility of 10 clinical Pseudomonas aeruginosa isolates to standard synthetic chemical antibiotics

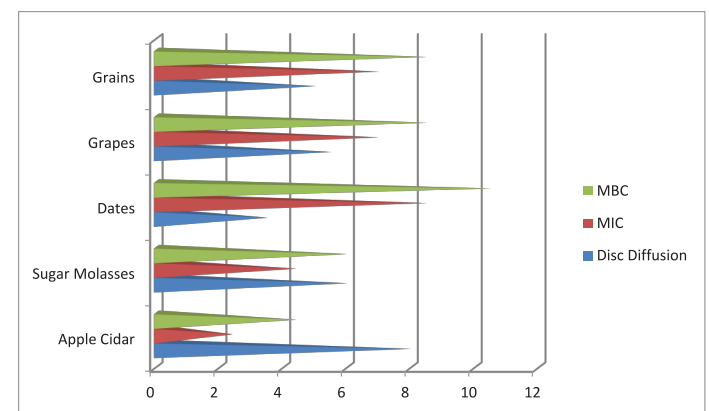

Fig. 2. Comparison of mean conventional susceptibility of 10 clinical Pseudomonas aeruginosa isolates to organic ethanoic acids derived from different sources.

Table 5. Comparison of minimum inhibitory concentrations of organic ethanoic acids among Pseudomonas aeruginosa isolates determined by conventional assays.

\begin{tabular}{lccccc}
\hline & \multicolumn{5}{c}{ Organic ethanoic acids $(\mu \mathrm{g} / \mathrm{mL})$} \\
\cline { 2 - 6 } Sample & Apple cider & Sugar molasses & Dates & Grapes & Grains \\
\hline Green pus & 0.5 & 0.75 & 1.25 & 1.25 & 1.25 \\
Green catheter & 0.5 & 0.5 & 1.25 & 1.25 & 1 \\
Urine & & & & & \\
& 0.5 & 0.75 & 1.25 & 1 & 1.25 \\
Greenish ventilator & 0.5 & 0.5 & 1.25 & 0.75 & 1.25 \\
Abscess & 0.5 & 0.75 & 1 & 0.75 & 1 \\
Throat swabs & 0.5 & 0.5 & 0.75 & 0.75 & 1.25 \\
Aspirates & 0.5 & 0.5 & 1 & 1.25 & 0.75 \\
Wound & 0.5 & 0.5 & 1 & 1 & 0.75 \\
Nasal swabs & 0.5 & 0.5 & 1.25 & 0.75 & 1.25 \\
Surgical & 0.5 & 0.1 & 1.5 & 1.25 & 0.75 \\
ATCC 27853 & 0.5 & 0.5 & 1 & 0.75 & 0.75 \\
Mean MIC $(\mu \mathrm{gg} / \mathrm{mL})$ & 0.5 & 0.75 & 1.25 & 1 & 1 \\
\hline
\end{tabular}

*For all samples. MIC, minimum inhibitory concentrations.

$P$. aeruginosa isolates. Table 5 shows a detailed comparison of the $\mathrm{MBC}$ of organic ethanoic acids for all the $P$. aeruginosa isolates.

The comparative findings of the antimicrobial susceptibility test values for the 10 clinical isolates of $P$. aeruginosa derived from patients with nosocomial infections suggests that organic ethanoic acid is significantly more effective than standard synthetic antibiotics.

\section{CONCLUSIONS}

The most important finding of the study was that the ATCC 27853 standard strain of $P$. aeruginosa was more susceptible to organic ethanoic acids than to standard synthetic antibiotics.
The susceptibility of the 10 clinical isolates of $P$. aeruginosa derived from the patients was variable compared with that to organic ethanoic acids. This finding indicates that the multidrugresistant strains in the clinical isolates might be the result of excessive antibiotic administration, especially during prolonged hospitalization. Multidrug-resistant strains emerge due to constant disregard for warnings about antibiotic use published by the World Health Organization (WHO). The present findings showed that natural remedies such as organic ethanoic acids can serve as an alternative to synthetic antibiotics not only for eradicating multidrug-resistant organisms but also for other infections, which together with zero side effects could help save many lives. 


\section{ABBREVIATIONS}

AK, amikacin; ATCC 27853- Standard Pseudomonas aeruginosa strain; GM, gentamicin; I, intermediate; MP, meropenem; $\mathrm{NI}$, nitrofurantoin; PM, cefepime; PTC, piperacillin/ tazobactam; R, resistant; S, sensitive; SZD, susceptibility zone diameter (mm); TC, tetracycline; TLC, ticarcillin/ clavulanic acid; TM, tobramycin; TS, trimethoprim; TX, ceftriaxone; TZ, ceftazidime.

\section{ACKNOWLEDGEMENTS}

We acknowledge Ibn Sina National College, Jeddah (Kingdom of Saudi Arabia) Administration for their constant support and encouragement due to which this project came to existence.

\section{AUTHOR CONTRIBUTIONS}

All authors listed have made a substantial, direct and intellectual contribution to the work, and approved it for publication.

\section{CONFLICT OF INTEREST}

The authors declare that there is no conflict of interest.

\section{FUNDING}

None.

\section{DATA AVAILABILITY}

All datasets generated or analyzed during this study are included in the manuscript and/or the Supplementary Files.

\section{ETHICS STATEMENT}

This article does not contain any studies with human participants or animals performed by any of the authors.

\section{REFERENCES}

1. Alaa S, Muazzam SM, Mohannad S. Role of Pseudomonas aeruginosa in nosocomial infection.
Int J Health Sci. 2018; 6(1):211-217.

2. Yetkin G, Otlu B, Cicek A, Kuzucu C, Durmaz R. Clinical, microbiologic, and epidemiologic characteristics of Pseudomonas aeruginosa infections in a University Hospital, Malatya, Turkey. Am J Infect Control. 2006;34(4):188-92. https://doi.org/10.1016/j. ajic.2005.11.010

3. Torabi S, Falak-ul-Aflaki B, Moezzi F. In vitro antimicrobial drug-resistance of urinary tract pathogens in patients admitted to Vali-e-Asr hospital wards. ZUMS J. 2007;15(61):79-88.

4. Lida B, Norkhoda S, Farid Azizi J, Hassan H, Sattar MD, Ali H, Sobhan G, Iraj P. E-test antibiotic susceptibility of E. coli strains isolated from hospital acquired infections of Imam Khomeini hospital, Ilam, Iran J Bas Res Med Sci. 2017;4(1):17-21. https://doi.org/10.18869/ acadpub.jbrms.4.1.17

5. Muazzam SM, Al Hasel HMB, Majid DH, Momen TN, Al Hazmi HAM, Al Jeddani FMS, Al Malaki RTW, Khan AA, Iqubal SMS. Orient J Chem. 2019;35(6):16951701.

6. Nagoba BS, Selkar SP, Wadher BJ, Gandhi RC. Acetic acid treatment of pseudomonal wound infections. J Infect Public Heal. 2013;6:410-415. https://doi. org/10.1016/j.jiph.2013.05.005

7. Luber P, Bartelt E, Genschow E, Wagner J, Hahn H. Comparison of broth microdilution, $\mathrm{E}$ test, and agar dilution methods for antibiotic susceptibility testing of Campylobacter jejuni and Campylobacter coli. J Clin Microbiol. 2003;41(3):1062-1068. https://doi. org/10.1128/JCM.41.3.1062-1068.2003

8. Nagoba BS, Deshmukh SR, Wadher BJ, Patil SB. Acetic acid treatment of pseudomonal postoperative wound infection. J Hosp Infect. 1997;36:243-244. https://doi. org/10.1016/S0195-6701(97)90201-2

9. Al-Ibran E, Khan M. Efficacy of topical application of $1 \%$ acetic acid in eradicating pseudomonal infections in burn wounds. J Dow Univ Health Sci. 2010;4:90-93.

10. Bagewadi ZK, Muddapur UM, Madiwal SS, Mulla SI, Khan AA. Biochemical and enzyme inhibitory attributes of methanolic leaf extract of Datura inoxia Mill. Environ Sustain. 2019;2:75. https://doi.org/10.1007/s42398019-00052-6

11. Gouse BS, Muazzam SM, Gokul SS, Ranjith MS. The efficacy of Cinnamomum tamala as a potential antimicrobial substance against the multi-drug resistant enterococcus faecalis from clinical isolates. Int J Pharm Pharmaceutical Sc. 2017;9(10):267.

12. Bisht CMS, Iqubal SMS, Khan AA, Tasneem M, Dawoud A, Gamal M, Singh SK, Asghar BH. Antifungal activity of Salvii Jordanii. J Pure Appl Microbiol. 2019; 13(3):1611. https://doi.org/10.22207/JPAM.13.3.34 